\title{
$p$-Nitrobenzoic Acid Promoted Synthesis of 1,5-Benzodiazepine Derivatives
}

\author{
Ravi Varala, * Ramu Enugala and Srinivas R. Adapa* \\ Indian Institute of Chemical Technology, Hyderabad-500 007, India
}

\begin{abstract}
Entre vários ácidos carboxílicos testados, o ácido $p$-nitrobenzóico mostrou ser o mais versátil para a preparação de derivados de 1,5-benzodiazepinas a partir de uma série de ofenilenodiaminas substituídas e cetonas. Os produtos foram obtidos em bons rendimentos (62$92 \%$ ) sob condições brandas, usando acetonitrila como solvente, a temperatura ambiente. Adicionalmente, o reagente pode ser facilmente recuperado e re-utilizado.

$p$-Nitrobenzoic acid was found to be the versatile Bronsted organic acid promoter among the carboxylic acids tested for the preparation of 1,5-benzodiazepine derivatives from a wide range of substituted o-phenylenediamines and ketones. The corresponding products were obtained in good isolated yields (62-92\%) under mild conditions using acetonitrile as solvent at ambient temperature. Further, the reagent could be easily recovered and reused.
\end{abstract}

Keywords: 1,5-benzodiazepines, bronsted acid, $o$-phenylenediamines and ketones, $p$-nitrobenzoic acid

\section{Introduction}

1,5-benzodiazepine derivatives have received significant attention and the core is indeed a "privileged scaffold" found in compounds active against a variety of target types including peptide hormones (such as CCK), interleukin converting enzymes (ICE) and potassium blockers $\left(\mathrm{I}_{\mathrm{k}}\right) \cdot{ }^{1}$ More recently, the area of biological interest of 1,5-benzodiazepines has been extended to various diseases such as cancer, viral infection (non-nucleoside inhibitors of HIV-1 reverse transcriptase), cardiovascular disorders. ${ }^{2}$ In addition, 1,5benzodiazepines show antidepressive, antifungal, antibacterial, antifeedant, antiinflammatory, analgesic and anticonvulsant activities. ${ }^{3}$ Besides these derivatives are also used as dyes for acrylic fibre in photography. ${ }^{4}$ Moreover, 1,5-benzodiazepines are valuable synthons used for the preparation of other fused ring compounds such as triazolo-, oxadiazolo-, oxazino-, or furano-benzodiazepines. ${ }^{5}$

Despite their importance from a pharmacological, industrial and synthetic point of view, comparatively few methods for their preparation are reported in the literature, a great number of which have appeared only very recently employing $\mathrm{BF}_{3}$-etherate, $\mathrm{NaBH}_{4}$, polyphosphoric acid or $\mathrm{SiO}_{2}, \mathrm{MgO} / \mathrm{POCl}_{3}, \mathrm{Yb}(\mathrm{OTf})_{3}$, and $\mathrm{InBr}_{3}$ as catalysts or as stoichiometric reagents. ${ }^{6}$ However, many of these methods

*e-mail: rvarala_iict@yahoo.co.in have some drawbacks such as low yields of the products, high temperatures, drastic reaction conditions, long reaction times, and relatively expensive reagents. From the viewpoints above, the development of environmentally benign, less expensive and easily handled promoters for the preparation of 1,5-benzodiazepines (hereafter 1,5BDPs) is still highly desirable.

Very recently, we have reported synthesis of 1,5-BDPs using CAN as catalyst in methanol. ${ }^{7}$ In recent years, lot of attention has been paid to organocatalysts owing to their eco-friendliness and can proceed under aerobic atmosphere, other notable advantages are: usually less expensive and commercially available. ${ }^{8}$ Intrigued by the results achieved by Zhao et al. ${ }^{9}$ in allylation of aldehydes using carboxylic acids as Bronsted organocatalysts and Gopal's report ${ }^{9}$ which was limited only for the reaction between acetone and $o$-phenelynediamine $(o$-PD) involving supramolecular chemistry, we have undertook advantage of both the reports and tried to explore the utility of several organo acids in the preparation of various structurally and electronically divergent 1,5-BDPs.

\section{Results and Discussion}

In continuation of our efforts to develop novel synthetic routes for carbon-carbon and carbon-heteroatom bond formations and heterocycles, ${ }^{10}$ we have studied the efficacy 
of chosen organoacids ( 1 equiv. as standard) for the model reaction using $o$-PD $(1 \mathrm{mmol})$ and acetophenone $(2.2$ $\mathrm{mmol}$ ) in acetonitrile at ambient temperature to afford the corresponding 1,5-benzodiazepine (Table 1).<smiles>[R]CC1([R])Nc2cc([R])c([R])cc2N=C([R])C1([R])C[R1]</smiles>

$\mathrm{R}, \mathrm{R}^{1}=\mathrm{H} ; \mathrm{R}=\mathrm{CH}_{3}, \mathrm{R}^{1}=\mathrm{H}$;

$\mathrm{R}=\mathrm{Cl}, \mathrm{R}^{1}=\mathrm{H} ; \mathrm{R}=\mathrm{NO}_{2}, \mathrm{R}^{1}=\mathrm{H}$;

$\mathrm{R}=\mathrm{CH}_{3}, \mathrm{R}^{1}=\mathrm{CH}_{3} ; \mathrm{R}=\mathrm{Cl}, \mathrm{R}^{1}=\mathrm{Cl}$

$\mathrm{R}=\mathrm{COPh}, \mathrm{R}^{1}=\mathrm{H}$

Scheme 1.

Among the acids screened, $p$-nitrobenzoic acid ( $p$ NBA) was found to be the best promoter in terms of reaction times and yields (entry 2, Table 1 ). To the best of our knowledge, there are no earlier reports on the preparation of 1,5-benzodiazepines using $p$-nitrobenzoic acid (Scheme 1). The optimum yields of the product were obtained when a ratio of $o$-PD to ketone 1:2.2 is used. The reaction is found to be sluggish when carried out using even 0.5 equiv. of catalyst amounts. In all cases, the reactions are clean and are completed within 3.5-15 h. The benzodiazepines were the only products obtained and the rest of the material was essentially starting material.

Table 1. Reaction of $o$-PD with acetophenone promoted by organoacids in 1,5-benzodiazepines synthesis

\begin{tabular}{llcc}
\hline Entry & Organo acid & time/h & Yield/(\%) \\
\hline 1 & benzoic acid & 24 & 80 \\
2 & $p$-nitrobenzoic acid & 07 & 90 \\
3 & $m$-bromobezoic acid & 24 & 60 \\
4 & phenylacetic acid & 24 & 80 \\
5 & mandelic acid & 24 & 32 \\
6 & $p$-tolunesulphonic acid & 72 & N.R \\
7 & cinnamic acid & 24 & N.R \\
8 & anthranilic acid & 24 & 83 \\
9 & $o$-picolinic acid & 24 & 79 \\
10 & isonicotinic acid & 24 & 83 \\
11 & malonic acid & 24 & 35 \\
12 & adipic acid & 12 & 78 \\
13 & valaric acid & 24 & 40 \\
14 & citric acid & 12 & 88 \\
15 & imino diacetic acid & 24 & N.R \\
16 & $p$-anisic acid & 24 & 85 \\
\hline
\end{tabular}

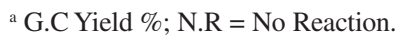

The crude product was filtered and washed with DCM to recover the $p$-nitrobenzoic acid, the filtrate was extracted with DCM and after usual workup and further purification by silica gel column chromatography yielded the desired pure product. Furthermore, $p$-NBA could be recovered and repeated minimum three times with slight decrease in the yield $(88 \%, 80 \%, 77 \%)$ due to the loss of $p$-NBA in recovery.
Encouraged by these results, at first, we studied the condensation of acetophenone with divergent $o$-PD's for the synthesis of corresponding 1,5-benzodiazepine derivatives under standardized conditions and the results were quite satisfactory (Table 2). The scope and generality of the present procedure was then extended to electronically divergent acetophenones towards $o$-PD and the results are presented in Table 3. It is interesting to note that under our optimized conditions, exclusively one regioisomer is favoured para position in relation to the imine moiety as confirmed by ${ }^{1} \mathrm{H}$ NMR spectroscopic data. (entries 2, 4, 6 and 7, Table 2).

Table 2. $p$-NBA mediated synthesis of 1,5-benzodiazepines from acetophenone

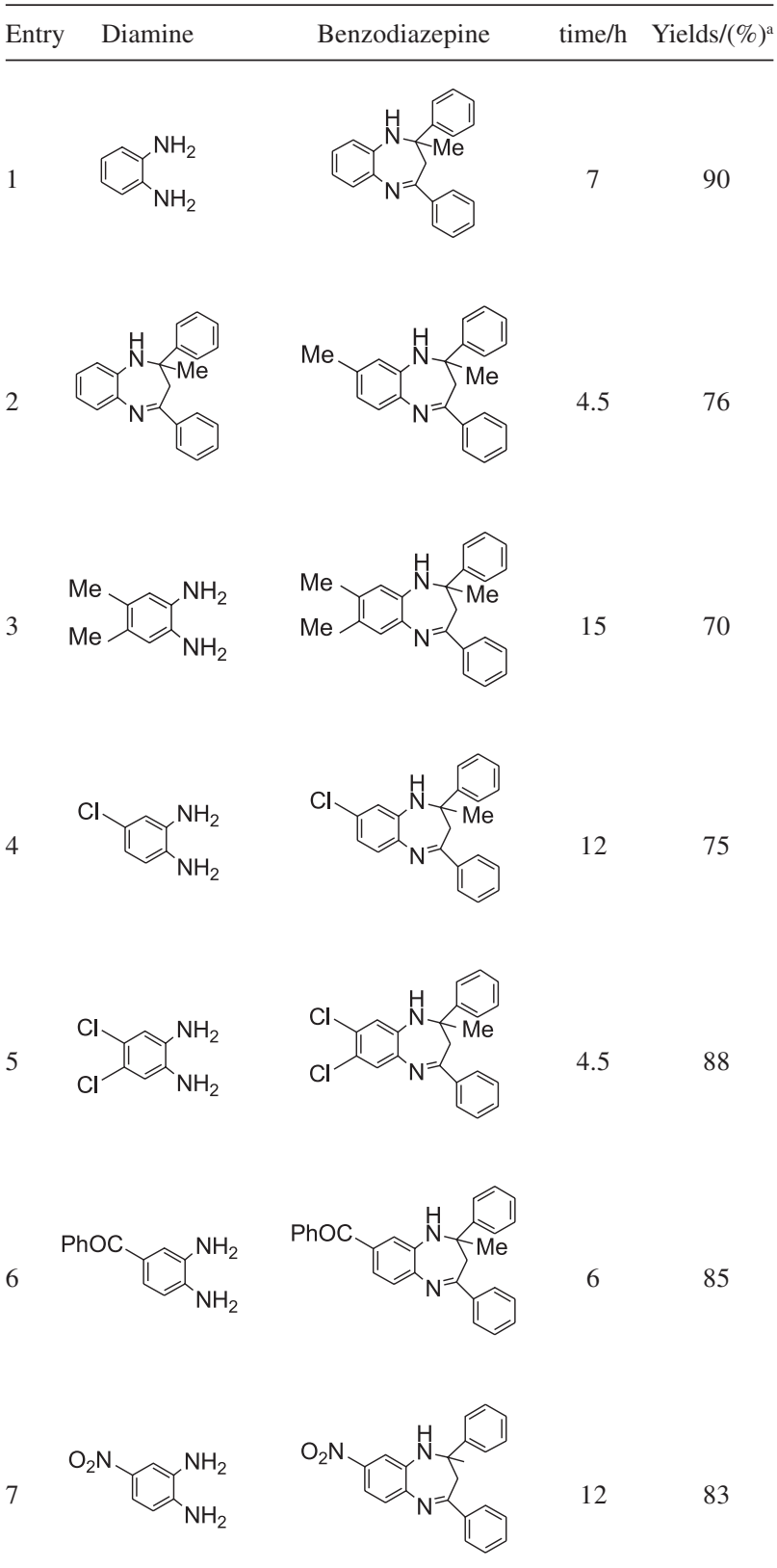

a Yields refer to the isolated pure products. 
In a similar fashion, cyclic ketones such as cyclopentanone and cyclohexanone also reacted well with $o$ PD to furnish the corresponding 1,5-BDPs in good yields (entries 6-7, Table 3). In case of unsymmetrical ketones such as 2-butanone (entry 8, Table 3), the ring closure occurred selectively giving a single product.

Table 3. $p$-NBA mediated synthesis of 1,5-benzodiazepines from $o$-PD

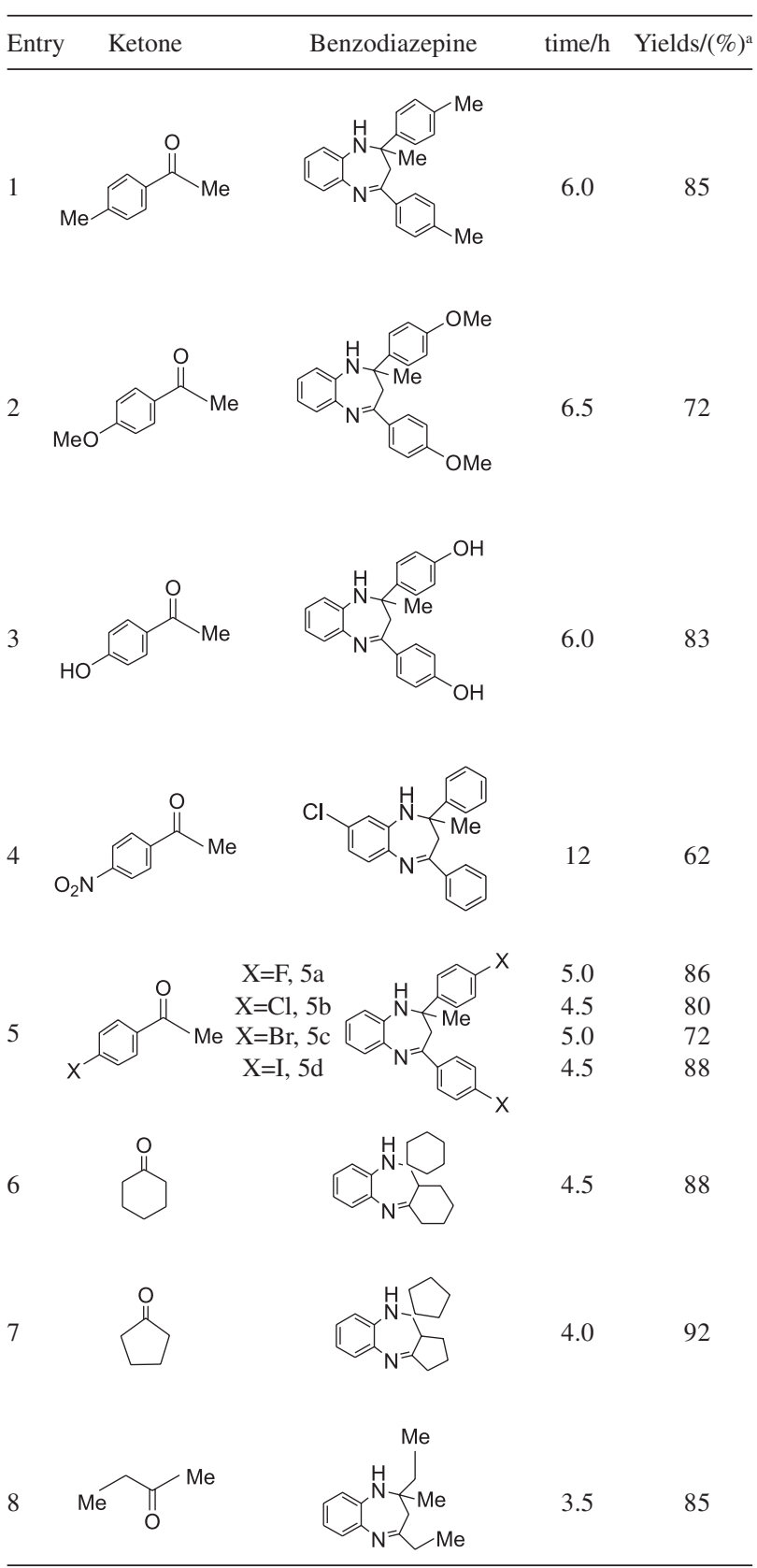

aYields refer to isolated pure products.

\section{Conclusions}

In conclusion, we have developed a practical and novel procedure for the synthesis of 2,3-dihydro- $1 \mathrm{H}-1,5$ - benzodiazepine derivatives using $p$-NBA in acetonitrile. The present protocol has several advantages: mild reaction conditions (at room temperature), operational and experimental simplicity, readily availability, study of wide range of electronically divergent ketones and $o$-PDs, easily recoverable and reusable organo promoter for applicability in large scale synthesis. We believe that this $p$-NBA promoted methodology will be a valuable contribution to the existing processes in the field of synthesis of 1,5benzodiazepines.

\section{Experimental}

\section{General}

Melting points were measured using a Buchi R-535 apparatus. IR spectra were taken on a Bruker Vector-22 spectrometer in $\mathrm{KBr}$ pellets and reported in $\mathrm{cm}^{-1}$. ${ }^{1} \mathrm{H} \mathrm{NMR}$ spectra were recorded at $300 \mathrm{MHz}$ on a Bruker Avance300 spectrometer in $\mathrm{CDCl}_{3}$ with chemical shifts $(\delta)$ given relative to TMS as an internal standard. ${ }^{13} \mathrm{C}$ NMR spectra were recorded on a Bruker Avance-300 (75.5 MHz) spectrometer with complete proton decoupling; chemical shifts are reported in ppm relative to the solvent resonance as the internal standard $\left(\mathrm{CDCl}_{3}, \delta 77.16 \mathrm{ppm}\right.$. $\mathrm{CHN}$ analyses were recorded on a Vario EL analyzer.

\section{Representative procedure}

To a suspension of $p$-nitrobenzoic acid $(1 \mathrm{mmol})$ in acetonitrile ( $3 \mathrm{~mL})$ were added successively $o$-phenylenediamine $(1 \mathrm{mmol})$ and acetophenone $(2.2$ mmol) at room temperature for the time specified in Table 2. After the reaction was over, the reaction mixture was filtered and washed with DCM to recover $p$-nitrobenzoic acid. The filtrate was extracted with DCM $(20 \mathrm{~mL})$ and the combined organic layer was dried over $\mathrm{Na}_{2} \mathrm{SO}_{4}$, concentrated under reduced pressure to furnish the crude product, which was purified by silica gel chromatography using EtOAc/Hexane (1:5), to afford corresponding pure product. All compounds gave satisfactory spectroscopic data in accordance to their proposed structures.

\section{Spectral data for compounds}

\section{Entry 1, Table 2}

Yellow solid. mp $150-152^{\circ} \mathrm{C}$. IR $(\mathrm{KBr}) v_{\max } / \mathrm{cm}^{-1}: 3320$, 1631, 1597. ${ }^{1} \mathrm{H}$ NMR (300 MHz, $\left.\mathrm{CDCl}_{3}\right) \delta: 1.80$ (s, 3H), 2.95 (d, 1H, J $12.8 \mathrm{~Hz}), 3.15$ (d, 1H, J $12.8 \mathrm{~Hz}) 3.45$ (br s, $\mathrm{NH}), 6.55-7.01(\mathrm{~m}, 3 \mathrm{H}), 7.15-7.35(\mathrm{~m}, 7 \mathrm{H}), 7.55-7.65(\mathrm{~m}$, $4 \mathrm{H}) ;{ }^{13} \mathrm{C} \mathrm{NMR}\left(75 \mathrm{MHz}, \mathrm{CDCl}_{3}\right) \delta: 29.7,42.9,73.3,121.2$, 
$121.4,125.2,126.1,126.8,126.9,127.8,128.1,128.5$, $129.5,137.9,139.5,139.9,147.4,167.3 . \mathrm{MS}(\mathrm{EI}), \mathrm{m} / \mathrm{z}$ $\left[\mathrm{M}^{+}\right]=312$. Anal. Calc. for $\mathrm{C}_{22} \mathrm{H}_{20} \mathrm{~N}_{2}: \mathrm{C}, 84.58 ; \mathrm{H}, 6.45 ; \mathrm{N}$, 8.97: Found: C, 84.55; H, 6.51; N, 8.94.

\section{Entry 2, Table 2}

Yellow solid. mp 92-94 ${ }^{\circ} \mathrm{C}$. IR ( KBr) $v_{\max } / \mathrm{cm}^{-1}: 3275$, 1659, 1614. ${ }^{1} \mathrm{H}$ NMR (300 MHz, $\left.\mathrm{CDCl}_{3}\right) \delta: 1.80$ (s, 3H), 2.41 (s, 3H), 3.01 (d, J 13.2 Hz, 1H), 3.15 (d, J $13.2 \mathrm{~Hz}$, 1H), 3.51 (br s, 1H, NH), 6.70-7.69 (m, 13H); ${ }^{13} \mathrm{C}$ NMR $\left(75 \mathrm{MHz}, \mathrm{CDCl}_{3}\right) \delta: 20.9,28.7,45.9,51.0,113.5,123.5$, $125.7,126.3,127.4,128.2,128.3,128.5,128.6,129.0$, 130.8, 131.2, 134.0, 136.9, 164.6. MS (EI), $\mathrm{m} / \mathrm{z}\left[\mathrm{M}^{+}\right]=$ 326. Anal. Calc. for $\mathrm{C}_{23} \mathrm{H}_{22} \mathrm{~N}_{2}: \mathrm{C}, 84.63 ; \mathrm{H}, 6.79 ; \mathrm{N}, 8.58$ : Found: C, 84.53; H, 6.85; N, 8.62.

\section{Entry 3, Table 2}

Yellow solid. mp 115-116 ${ }^{\circ} \mathrm{C}$. IR $(\mathrm{KBr}) \mathrm{v}_{\max } / \mathrm{cm}^{-1}$ : 3285, 1635, 1609. ' $\mathrm{H}$ NMR (300 MHz, $\left.\mathrm{CDCl}_{3}\right) \delta: 1.70$ (s, $3 \mathrm{H}), 2.25$ (s, 6H), 2.90 (d, 1H, J $12.8 \mathrm{~Hz}), 3.10$ (d, 1H, J $12.8 \mathrm{~Hz}), 3.45$ (br s, 1H), 6.60 (s, 1H), 7.15 (s, 1H), 7.18$7.30(\mathrm{~m}, 6 \mathrm{H}), 7.50-7.60(\mathrm{~m}, 4 \mathrm{H}) ;{ }^{13} \mathrm{C} \mathrm{NMR}(75 \mathrm{MHz}$, $\left.\mathrm{CDCl}_{3}\right) \delta: 18.6,19.3,29.7,43.2,73.0,122.3,125.4,126.8$, 126.9, 127.8, 128.2, 129.3, 129.4, 129.6, 134.8, 135.7, 137.6, 139.7, 147.8, 166.8. MS (EI), $m / z\left[\mathrm{M}^{+}\right]=340$. Anal. Calc. for $\mathrm{C}_{24} \mathrm{H}_{24} \mathrm{~N}_{2}$ : C, 84.66; H, 7.10; N, 8.22: Found: C, 84.78; H, 7.25; N, 8.35.

\section{Entry 4, Table 2}

Yellow solid. mp $121-123{ }^{\circ} \mathrm{C}$. IR $(\mathrm{KBr}) \mathrm{v}_{\max } / \mathrm{cm}^{-1}$ : 3290, 1647, 1598, 854. ${ }^{1} \mathrm{H}$ NMR (300 MHz, $\left.\mathrm{CDCl}_{3}\right)$ $\delta: 1.76(\mathrm{~s}, 3 \mathrm{H}), 2.96(\mathrm{~d}, J 12.8 \mathrm{~Hz}, 1 \mathrm{H}), 3.15(\mathrm{~d}, J 12.8$ $\mathrm{Hz}, 1 \mathrm{H}), 3.35$ (br s, NH), 6.78-6.81 (m, 1H), 6.95-6.98 $(\mathrm{m}, 1 \mathrm{H}), 7.15-7.27(\mathrm{~m}, 6 \mathrm{H}), 7.42-7.58(\mathrm{~m}, 3 \mathrm{H}), 7.91-7.96$ $(\mathrm{m}, 2 \mathrm{H}) ;{ }^{13} \mathrm{C} \mathrm{NMR}\left(75 \mathrm{MHz}, \mathrm{CDCl}_{3}\right) \delta: 29.6,44.7,71.2$, $120.9,122.9,124.1,128.9,139.9,130.5$, 132.0, 137.6, 138.2, 146.0, 169.1. MS (EI), $m / z\left[\mathrm{M}^{+}\right]=346$. Anal. Calc. for $\mathrm{C}_{22} \mathrm{H}_{19} \mathrm{ClN}_{2}$ : C, 76.18; H, 5.52; N, 8.08: Found: C, 76.07; H, 5.49; N, 8.12.

\section{Entry 5, Table 2}

Yellow crystalline solid. mp $158-160{ }^{\circ} \mathrm{C}$. IR (KBr) $v_{\max } / \mathrm{cm}^{-1}: 3304,1638,1603,761 .{ }^{1} \mathrm{H}$ NMR $(300 \mathrm{MHz}$, $\left.\mathrm{CDCl}_{3}\right) \delta: 1.75$ (s, $\left.3 \mathrm{H}\right), 2.93$ (d, J $\left.12.8 \mathrm{~Hz}, 1 \mathrm{H}\right), 3.12$ (d, $J 12.8 \mathrm{~Hz}, 1 \mathrm{H}$ ), 3.49 (br s, NH), 6.88 (s, $1 \mathrm{H}$ ), 7.13-7.30 $(\mathrm{m}, 5 \mathrm{H}), 7.36$ (s, 1H), 7.50-7.53 (m, $5 \mathrm{H}) ;{ }^{13} \mathrm{C}$ NMR $(75$ $\left.\mathrm{MHz} \mathrm{CDCl}_{3}\right) \delta: 29.9,43.2,72.9,121.9,124.1,125.3$ 127.3, 128.4, 128.7, 130.1, 137.8, 139.3, 146.8, 168.8. MS (EI), $m / z\left[\mathrm{M}^{+}\right]=381$. Anal. Calc. For $\mathrm{C}_{22} \mathrm{H}_{18} \mathrm{Cl}_{2} \mathrm{~N}_{2}: \mathrm{C}$, 69.30; H, 4.76; N, 7.35: Found: C, 69.28; H, 4.81; N, 7.32 .

\section{Entry 6, Table 2}

Yellow solid. mp $114-116{ }^{\circ} \mathrm{C}$. IR $(\mathrm{KBr}) v_{\max } / \mathrm{cm}^{-1}$ : 3327, 1681, 1649, 1601, 1249, 1178, 981, 820. ${ }^{1} \mathrm{H}$ NMR $\left(300 \mathrm{MHz}, \mathrm{CDCl}_{3}\right) \delta: 1.78(\mathrm{~s}, 3 \mathrm{H}), 2.96(\mathrm{~d}, 1 \mathrm{H}, J 12.8$ $\mathrm{Hz}$ ), 3.31 (d, 1H, J 12.8 Hz), 3.51 (br s, 1H, NH), 7.12$7.26(\mathrm{~m}, 5 \mathrm{H}), 7.34-7.61(\mathrm{~m}, 7 \mathrm{H}), 7.72-7.78(\mathrm{~m}, 2 \mathrm{H})$, 7.81-7.85 (m, 1H), 7.88-7.92 (m, 2H), 7.98-8.01 (m, $1 \mathrm{H}) ; \mathrm{MS}(\mathrm{FAB}), \mathrm{m} / \mathrm{z}\left[\mathrm{M}^{+}+\mathrm{H}\right]=417$. Anal. Calc. for $\mathrm{C}_{29} \mathrm{H}_{24} \mathrm{~N}_{2} \mathrm{O}$ : C, 83.63; H, 5.81; N, 6.73; Found: C, 83.49; H, 5.94; N, 6.69 .

\section{Entry 7, Table 2}

Yellow solid. mp $136-138{ }^{\circ} \mathrm{C}$. IR $(\mathrm{KBr}) v_{\max } / \mathrm{cm}^{-1}$ : 3318, 1638, 1554, 1356. ${ }^{1} \mathrm{H}$ NMR (300 $\mathrm{MHz}, \mathrm{CDCl}_{3}$ ) $\delta: 1.79(\mathrm{~s}, 3 \mathrm{H}), 3.06(\mathrm{~d}, 1 \mathrm{H}, J 13.4 \mathrm{~Hz}), 3.32(\mathrm{~d}, 1 \mathrm{H}, J$ $13.4 \mathrm{~Hz}$ ), 4.41 (br s, 1H, NH), 6.92-6.94 (d, 1H, J 8.7 $\mathrm{Hz}), 7.17-7.55(\mathrm{~m}, 10 \mathrm{H}), 7.92-7.96(\mathrm{~m}, 2 \mathrm{H}) ;{ }^{13} \mathrm{C} \mathrm{NMR}$ $\left(75 \mathrm{MHz}, \mathrm{CDCl}_{3}\right) \delta: 29.1,45.8,65.8,111.8,116.9,124.2$, $125.8,127.6,128.8,129.2,132.2,132.5,133.4,135.6$, 137.8, 139.6, 143.1, 166.8. MS (EI), $m / z\left[\mathrm{M}^{+}\right]=357$. Anal. Calc. for $\mathrm{C}_{22} \mathrm{H}_{19} \mathrm{~N}_{3} \mathrm{O}_{2}$ : C, 73.93; H, 5.36; N, 11.76; Found: C, 73.87; H, 5.41; N, 11.67.

\section{Entry 1, Table 3}

Pale yellow crystalline solid. mp 98-99 ${ }^{\circ} \mathrm{C}$. IR (KBr) $\mathrm{v}_{\max } / \mathrm{cm}^{-1}: 3318,1630,1598 .{ }^{1} \mathrm{H}$ NMR $\left(300 \mathrm{MHz}, \mathrm{CDCl}_{3}\right)$ $\delta: 1.72(\mathrm{~s}, 3 \mathrm{H}), 2.26(\mathrm{~s}, 3 \mathrm{H}), 2.32$ (s, 3H), 2.98 (d, 1H, J $13.4 \mathrm{~Hz}$ ), 3.05 (d, 1H, J $13.4 \mathrm{~Hz}$ ), 3.43 (br s, 1H, NH), 6.74-6.76 (m, 1H), 6.98-7.02 (m, 6H), 7.21-7.23 (m, 1H), 7.47-7.50 (m, 4H); $\left.{ }^{13} \mathrm{C} \mathrm{NMR} \mathrm{(75} \mathrm{MHz}, \mathrm{CDCl}_{3}\right) \delta: 20.7$, 21.2, 29.8, 42.9, 73.1, 121.3, 121.4, 125.2, 126.0, 127.1, $128.5,128.6,128.9,129.2,136.4,137.0,138.2$, 139.7, 140.3, 145.0, 166.8. MS (EI), $\mathrm{m} / z\left[\mathrm{M}^{+}\right]=340$. Anal. Calc. for $\mathrm{C}_{24} \mathrm{H}_{24} \mathrm{~N}_{2}$ : C, 84.70; H, 7.05; N, 8.23: Found: C, 84.68; $\mathrm{H}, 7.13 ; \mathrm{N}, 8.18$.

\section{Entry 2, Table 3}

Yellowish solid. mp 114-116 ${ }^{\circ} \mathrm{C}$. IR (neat) $\mathrm{v}_{\max } / \mathrm{cm}^{-1}$ : 3325, 1135, 1640, 1594, 1190. ${ }^{1} \mathrm{H}$ NMR (200 MHz, $\left.\mathrm{CDCl}_{3}\right) \delta: 1.71(\mathrm{~s}, 3 \mathrm{H}), 2.85(\mathrm{~d}, 1 \mathrm{H}, J 12.8 \mathrm{~Hz}), 2.98$ (d, $1 \mathrm{H}, J 12.8 \mathrm{~Hz}), 3.73$ (s, 3H), 3.77 (s, 3H), 6.65-6.78 (m, $4 \mathrm{H}), 6.95-7.02(\mathrm{~m}, 2 \mathrm{H}), 7.18-7.25(\mathrm{~m}, 2 \mathrm{H}), 7.42-7.55(\mathrm{~m}$, $4 \mathrm{H}) ; \mathrm{MS}(\mathrm{EI}), m / z\left[\mathrm{M}^{+}\right]=372$. Anal. Calc. for $\mathrm{C}_{24} \mathrm{H}_{24} \mathrm{~N}_{2} \mathrm{O}_{2}$ : C, 77.37; H, 6.52; N, 7.50: Found: C, 77.18; H, 6.64; N, 7.52 .

\section{Entry 3, Table 3}

Yellow crystalline solid. mp 219-220 ${ }^{\circ} \mathrm{C}$. IR (KBr) $v_{\max } / \mathrm{cm}^{-1}: 3339,1636,1599 .{ }^{1} \mathrm{H}$ NMR $\left(200 \mathrm{MHz}, \mathrm{CDCl}_{3}\right)$ $\delta: 1.65$ (s, 3H), $2.77(\mathrm{~d}, 1 \mathrm{H}, J 12.6 \mathrm{~Hz}), 2.89$ (d, 1H, J $12.6 \mathrm{~Hz}$ ), 4.18 (br s, NH), 6.57-6.64 (m, 4H), 6.81-7.00 
(m, 1H), 7.10-7.18 (m, 1H), 7.28-7.55 (m, 4H). ${ }^{13} \mathrm{C}$ NMR $\left(75 \mathrm{MHz} \mathrm{CDCl}_{3}\right) \delta: 29.2,42.0,72.6,114.4,114.6,120.5$, $120.9,124.9,125.8,127.0,128.2,130.0,137.9,139.5$, 155.3, 158.6, 166.8. MS (EI), $m / z\left[\mathrm{M}^{+}\right]=344$. Anal. Calc. for $\mathrm{C}_{22} \mathrm{H}_{20} \mathrm{~N}_{2} \mathrm{O}_{2}$ : C, 76.74; $\mathrm{H}, 5.81 ; \mathrm{N}, 8.15$ : Found: $\mathrm{C}$, 76.76; H, 5.84; N, 8.13.

\section{Entry 4, Table 3}

Red crystalline solid. mp $156-158{ }^{\circ} \mathrm{C}$. IR (KBr) $v_{\max }$ ' $\mathrm{cm}^{-1}: 3325,1642,1597 .{ }^{1} \mathrm{H}$ NMR $\left(300 \mathrm{MHz}, \mathrm{CDCl}_{3}\right)$ $\delta: 1.83(\mathrm{~s}, 3 \mathrm{H}), 2.96(\mathrm{~d}, 1 \mathrm{H}, J 13.4 \mathrm{~Hz}), 3.27(\mathrm{~d}, 1 \mathrm{H}, J$ $13.4 \mathrm{~Hz}), 3.52$ (br s, NH), 6.97-6.98 (m, 1H), 7.00-7.02 (m, 6H), 7.21-7.22 (m, 1H), 7.45-7.47 (m, 4H); ${ }^{13} \mathrm{C} \mathrm{NMR}$ $\left(75 \mathrm{MHz} \mathrm{CDCl}_{3}\right) \delta: 30.0,42.9,73.4,121.3,122.2,123.5$, 123.4, 126.8, 127.6, 127.7, 129.6, 137.2, 138.8, 144.6, 146.9, 148.4, 154.1, 163.8. MS (EI), $m / z\left[\mathrm{M}^{+}\right]=402$. Anal. Calc. for $\mathrm{C}_{22} \mathrm{H}_{18} \mathrm{~N}_{4} \mathrm{O}_{4}$ : C, 65.67; H, 4.47; N, 13.93: Found: C, 65.65; H, 4.40; N, 13.98 .

\section{Entry 5a, Table 3}

Pale yellow crystalline solid. mp $104-105^{\circ} \mathrm{C}$. IR (KBr) $v_{\max } / \mathrm{cm}^{-1}: 3271,1651,1603,1231 .{ }^{1} \mathrm{H}$ NMR $(300 \mathrm{MHz}$, $\left.\mathrm{CDCl}_{3}\right) \delta: 1.75(\mathrm{~s}, 3 \mathrm{H}), 2.87(\mathrm{~d}, 1 \mathrm{H}, J 13.6 \mathrm{~Hz}), 3.04$ (d, $1 \mathrm{H}, J 13.6 \mathrm{~Hz}), 3.30$ (br s, NH), 6.75-6.79 (m, 1H), 6.82$6.92(\mathrm{~m}, 4 \mathrm{H}), 7.00-7.05(\mathrm{~m}, 2 \mathrm{H}), 7.19-7.25(\mathrm{~m}, 1 \mathrm{H}), 7.48-$ $7.62(\mathrm{~m}, 4 \mathrm{H}) ;{ }^{13} \mathrm{C} \mathrm{NMR}\left(75 \mathrm{MHz}, \mathrm{CDCl}_{3}\right) \delta: 29.7,42.9$, $73.4,114.7,114.8,115.0,115.1,126.2,127.0,128.5$, $129.2,135.5,137.4,140.3,143.2,160.2,162.2,165.3$, 165.5, 163.5. MS (EI), $m / z\left[\mathrm{M}^{+}\right]=348$. Anal. Calc. for $\mathrm{C}_{22} \mathrm{H}_{18} \mathrm{~F}_{2} \mathrm{~N}_{2}$ : C, 75.86; H, 5.17; N, 8.04: Found: C, 75.82; H, 5.20; N, 8.07.

\section{Entry 5b, Table 3}

Pale yellow crystalline solid. mp $143-145^{\circ} \mathrm{C}$. IR (KBr) $v_{\max } / \mathrm{cm}^{-1}: 3269,1636,1593,765 .{ }^{1} \mathrm{H}$ NMR $(200 \mathrm{MHz}$, $\left.\mathrm{CDCl}_{3}\right) \delta: 1.70(\mathrm{~s}, 3 \mathrm{H}), 2.79$ (d, $\left.1 \mathrm{H}, J 13.3 \mathrm{~Hz}\right), 2.97$ (d, $1 \mathrm{H}, J 13.3 \mathrm{~Hz}$ ), 3.25 (br s, NH), 6.68-6.75 (m, 1H), 6.92$7.02(\mathrm{~m}, 1 \mathrm{H}), 7.12-7.20(\mathrm{~m}, 5 \mathrm{H}), 7.38-7.52(\mathrm{~m}, 5 \mathrm{H}) ;{ }^{13} \mathrm{C}$ NMR (75 MHz, $\left.\mathrm{CDCl}_{3}\right) \delta: 29.7,42.8,73.4,121.4,121.9$, $126.6,127.0,128.2$, 128.3, 128.6, 133.0, 137.5, 137.7, 139.8, 145.8, 165.9. MS (EI), $\mathrm{m} / z\left[\mathrm{M}^{+}\right]=381$. Anal. Calc. for $\mathrm{C}_{22} \mathrm{H}_{18} \mathrm{Cl}_{2} \mathrm{~N}_{2}$ : C, 69.29; H, 4.72; N, 7.34: Found: C, 69.30; H, 4.78; N, 7.31.

\section{Entry 5c, Table 3}

Yellow solid. mp 145-146 ${ }^{\circ} \mathrm{C}$. IR $(\mathrm{KBr}) \mathrm{v}_{\max } / \mathrm{cm}^{-1}$ : 3325, 1640, 1589, 1198, 574. ${ }^{1} \mathrm{H}$ NMR (200 MHz, $\left.\mathrm{CDCl}_{3}\right) \delta: 1.72(\mathrm{~s}, 3 \mathrm{H}), 2.87(\mathrm{~d}, 1 \mathrm{H}, J 13.6 \mathrm{~Hz}), 3.00$ (d, 1H, J 13.6 Hz), 2.65 (br s, NH), 6.97-6.98 (m, 1H), 7.00-7.04 (m, 6H), 7.18-7.24 (m, 1H), 7.45-7.48 (m, $4 \mathrm{H})$. MS $(\mathrm{FAB}), \mathrm{m} / z\left[\mathrm{M}^{+}+\mathrm{H}\right]=471$. Anal. Calc. for
$\mathrm{C}_{22} \mathrm{H}_{18} \mathrm{Br}_{2} \mathrm{~N}_{2}: \mathrm{C}, 56.17 ; \mathrm{H}, 3.82 ; \mathrm{N}, 5.95$ : Found: C, $56.19 ; \mathrm{H}, 3.78 ; \mathrm{N}, 5.89$.

\section{Entry 5d, Table 3}

Pale yellow crystalline solid. mp $143-144{ }^{\circ} \mathrm{C}$. IR (KBr) $v_{\max } / \mathrm{cm}^{-1}: 3259,1636,1579,462 .{ }^{1} \mathrm{H}$ NMR $(200 \mathrm{MHz}$, $\mathrm{CDCl}_{3}$ ) $\delta: 1.71$ (s, 3H), 2.85 (d, 1H, J $\left.12.8 \mathrm{~Hz}\right), 2.99$ (d, $1 \mathrm{H}, J 12.8 \mathrm{~Hz}$ ), 3.32 (br s, NH), 6.73-6.75 (m, 1H), 6.98$7.03(\mathrm{~m}, 2 \mathrm{H}), 7.21-7.33(\mathrm{~m}, 5 \mathrm{H}), 7.53-7.58(\mathrm{~m}, 4 \mathrm{H}) ;{ }^{13} \mathrm{C}$ NMR (75 MHz, $\left.\mathrm{CDCl}_{3}\right) \delta: 30.3,43.4,74.1,93.4,97.4$, $122.1,122.6,127.3,128.2,129.2,129.3,137.8,138.0$, 139.4, 140.4, 147.6, 166.8. MS (FAB), $m / z\left[\mathrm{M}^{+}+\mathrm{H}\right]=565$. Anal. Calc. for $\mathrm{C}_{22} \mathrm{H}_{18} \mathrm{I}_{2} \mathrm{~N}_{2}$ : C, 46.97; H, 3.20; N, 4.98: Found: C, 46.92; H, 3.22; N, 5.01.

\section{Entry 6, Table 3}

Yellow solid. mp 137-138 ${ }^{\circ} \mathrm{C}$. IR $(\mathrm{KBr}) v_{\max } / \mathrm{cm}^{-1}$ : 3290, 1640, 1600. ${ }^{1} \mathrm{H}$ NMR (200 MHz, $\left.\mathrm{CDCl}_{3}\right) \delta: 1.23-$ 1.85 (m, 16H), 2.30-2.70 (m, 3H), 4.49 (br s, 1H), 6.65$7.35(\mathrm{~m}, 4 \mathrm{H}) ;{ }^{13} \mathrm{C} \mathrm{NMR}\left(75 \mathrm{MHz}, \mathrm{CDCl}_{3}\right) \delta: 21.6,21.7$, 23.2, 24.5, 25.3, 33.2, 34.4, 39.3, 40.5, 52.4, 63.1, 121.3, 121.5, 126.3, 129.6, 138.1, 142.6, 178.9. MS (EI), $\mathrm{m} / \mathrm{z}$ $\left[\mathrm{M}^{+}\right]=268$. Anal. Calc. for $\mathrm{C}_{18} \mathrm{H}_{24} \mathrm{~N}_{2}$ : C, 80.55; H, 9.01; N, 10.44: Found: C, 80.26; H, 9.54; N, 10.31 .

\section{Entry 7, Table 3}

Yellow solid. mp $138-140{ }^{\circ} \mathrm{C}$. IR $(\mathrm{KBr}) v_{\max } / \mathrm{cm}^{-1}$ : 3338, 1659, 1600. ${ }^{1} \mathrm{H}$ NMR (200 $\left.\mathrm{MHz}, \mathrm{CDCl}_{3}\right) \delta: 1.30-$ 1.90 (m, 12H), 2.30-2.61 (m, 3H), 4.50 (br s, 1H), 6.70$7.39(\mathrm{~m}, 4 \mathrm{H}) ;{ }^{13} \mathrm{C} \mathrm{NMR}\left(75 \mathrm{MHz}, \mathrm{CDCl}_{3}\right) \delta: 23.4,24.1$, 24.3, 28.7, 33.4, 38.5, 39.2, 54.4, 67.3, 118.6, 119.3, 126.9, 132.1, 139.2, 143.4, 178.0. MS (EI), $\mathrm{m} / \mathrm{z}\left[\mathrm{M}^{+}\right]=240$. Anal. Calc. for $\mathrm{C}_{16} \mathrm{H}_{20} \mathrm{~N}_{2}$ : C, 79.96; H, 8.39; N, 11.66: Found: C, 79.64; H, 8.22; N, 11.45 .

\section{Entry 8, Table 3}

Yellow solid. mp 137-139 ${ }^{\circ} \mathrm{C}$. IR $(\mathrm{KBr}) v_{\max } / \mathrm{cm}^{-1}$ : 3329, 1637, 16057. ${ }^{1} \mathrm{H}$ NMR (200 MHz, $\mathrm{CDCl}_{3}$ ) $\delta: 0.99$ (t, J 6.9 Hz, 3H), 1.25 (t, J $6.9 \mathrm{~Hz}, 3 \mathrm{H}), 1.70(\mathrm{q}, J 6.9 \mathrm{~Hz}$, 2H), 2.15 (m, 2H), 2.35 (s, 3H), 2.69 (q, J 6.9 Hz, 2H), 3.27 (br s, $1 \mathrm{H}, \mathrm{NH}), 6.78-7.35(\mathrm{~m}, 4 \mathrm{H}) ;{ }^{13} \mathrm{C}$ NMR $(75$ $\left.\mathrm{MHz}, \mathrm{CDCl}_{3}\right) \delta: 8.7,10.8,26.9,35.5,35.7,42.1,70.5$, 121.8, 125.4, 126.2, 127.0, 137.9, 140.8, 175.6. MS (FAB), $m / z\left[\mathrm{M}^{+}+\mathrm{H}\right]=216$. Anal. Calc. for $\mathrm{C}_{14} \mathrm{H}_{20} \mathrm{~N}_{2}: \mathrm{C}, 77.73 ; \mathrm{H}$, 9.32; N, 12.95: Found: C, 77.71; H, 9.36; N, 12.93.

\section{Acknowledgments}

Ravi Varala thanks DIICT, Dr. J. S. Yadav and Council of Scientific Industrial Research (CSIR, India) for financial support. 


\section{References}

1. Herpin, T. F.; Van Kirk, K. G.; Salvino, J. M.; Yu, S. T.; Labaudinière, R. F.; J. Comb. Chem. 2000, 5, 513.

2. Atwal, K. S.; Bergey, J. L.; Hedberg, A.; Moreland, S.; J. Med. Chem. 1987, 30, 635; Merluzzi, V.; Hargrave, K. D.; Labadia, M.; Grozinger, K.; Skoog, M.; Wu, J. C.; Shih, C-K; Eckner, K.; Hattox, S.; Adams, J.; Rosenthal, A. S.; Faanes, R.; Eckner, R. J.; Koup R. A.; Sullivan, J. L.; Science 1990, 250, 1411; Di Braccio, M.; Grossi, G.; Roma, G.; Vargiu, L.; Mura, M.; Marongiu, M. E.; Eur. J. Med. Chem. 2001, 36, 935.

3. Schutz, H.; Benzodiazepines, Springer: Heidelberg, 1982; Landquist, J. K. In Comprehensive Heterocyclic Chemistry; Katritzky, A. R.; Rees, C. W., eds. Pergamon: Oxford, 1984, vol. 1. pp.166-170; Randall, L. O.; Kappel, B. In Benzodiazepines, Garattini, S.; Mussini, E.; Randall, L. O.; eds., Raven Press: New York, 1973, p. 27

4. Haris, R. C.; Straley, J. M.; U. S. Patent $1,537,757$, 1968. (CA 1970, 73, 100054w)

5. El-Sayed, A. M. El.; Abdel-Ghany, H.; El-Saghier, A. M. M.; Synth. Commun. 1999, 29, 3561; Xu, J. X.; Wu, H. T.; Jin, S.; Chin. J. Chem. 1999, 17, 84; Zhang, X. Y.; Xu, J. X.; Jin, S.; Chin. J. Chem. 1999, 17, 404; Kim, K.; Volkman, S. K.; Ellman, J. A.; J. Braz. Chem. Soc. 1998, 9, 375.
6. Herbert, J. A. L.; Suschitzky, H.; J. Chem. Soc., Perkin Trans. 1 1974, 2657; Morales, H. R.; Ulbarela, B. A.; Contreras, R.; Heterocycles 1986, 24, 135; Jung, D. I.; Choi, T. W.; Kim, Y. Y.; Kim, I. S.; Park, Y. M.; Lee, Y. G.; Jung, D. H.; Synth. Commun. 1999, 29, 1941; Balakrishna, M. S.; Kaboudin, B.; Tetrahedron Lett. 2001, 42, 1127; Curini, M.; Epifano, F.; Marcotullio, M. C.; Rosati, O.; Tetrahedron Lett. 2001, 42, 3193; Yadav, J. S.; Reddy, B. V. S.; Kumar, S. P.; Nagaiah, K.; Synthesis 2005, 3, 480 and references cited therein.

7. Varala, R.; Ramu, E.; Sreelatha, N.; Adapa, S. R.; Synlett 2006, 7, 1009.

8. Peter, P. I. D.; Moisan, L.; Angew. Chem., Int. Ed. 2001, 40, 3726.

9. Li, Gui-long.; Zhao, G.; J. Org. Chem. 2005, 70, 4272; Thakuria, H.; Pramanik, A.; Borah, B. M.; Das, G.; Tetrahedron Lett. 2006, 47, 3135; Yanagisawa, A.; Nakamura, Y.; Arai, T.; Tetrahedron: Asymmetry 2004, 15, 1909.

10. Varala, R.; Ramu, E.; Sreelatha, N.; Adapa, S. R.; Tetrahedron Lett. 2006, 47, 877; Varala, R.; Adapa, S. R.; Org. Process Res. Dev. 2005, 9, 853; Varala, R.; Sreelatha, N.; Adapa, S. R.; Synlett 2006, 10, 1549 and references cited therein; Varala, R.; Ramu, E.; Adapa, S. R.; Synthesis 2006, 3825.

Received: July 8, 2006 Web Release Date: February 15, 2007 